Ann. Biol. anim. Bioch. Biophys., I972, 12 (2), 22I-23I.

\title{
CONTRÔLE DE L'ÉVOLUTION DE L'ÉQUIPEMENT ENZYMATIQUE DU PANCRÉAS EXOCRINE DU LAPIN DE LA NAISSANCE A 6 SEMAINES
}

\author{
T. CORRING, F. LEBAS et D. COURTOT * ${ }^{(1)}$
}

avec la collaboration technique de Anne-Marie Guevgneav, G. SARDi et J.-J. Baudet

Station de Recherches sur l'Élevage des Porcs, Centre nationale de Recherches zootechniques, I. N. R. A., 78 - Jouy-en-Josas

* Département de Biologie, Institut national des Sciences appliquées, 69 - Villeurbanne

\section{RÉSUMÉ}

Dans le but de vérifier, chez le lapereau sous la mère, l'hypothèse d'une adaptation du pancréas exocrine aux variations du régime alimentaire au moment du sevrage, 2 lots de 57 lapins ont été affectés aux régimes expérimentaux suivants :

I. allaitement maternel exclusif entre la naissance et le $3^{\circ}$ jour puis sevrage brutal avec un aliment amylacé ;

2. allaitement maternel exclusif entre la naissance et le $2 \mathbf{I}^{\mathbf{e}}$ jour puis sevrage brutal avec le même aliment amylacé.

Les activités enzymatiques de la trypsine, la chymotrypsine, la lipase et l'amylase ont été déterminées à 9 stades répartis entre 7 et 43 jours. Les résultats montrent que, quel que soit le régime expérimental, les activités enzymatiques présentent toutes une augmentation vers le $2 \mathbf{I}-24^{\mathrm{e}}$ jour, indépendante du poids des animaux. Ces activités sont cependant légèrement supérieures chez les animaux sevrés à 21 jours. Ceci semble indiquer que l'aliment n'est pas le facteur stimulant le développement des enzymes pancréatiques. Mais il serait susceptible de moduler l'ampleur de la réponse à cette stimulation.

(1) Adresse actuelle : Laboratoire de Pharmacologie-Toxicologie, I. N. R. A., I80 chemin de Tournefeuille, $3 \mathrm{I}$ - Toulouse 03 . 


\section{INTRODUCTION}

Les observations concernant l'activité de certaines enzymes du pancréas exocrine à certains stades du développement de l'animal mettent en évidence deux faits principaux :

- les activités enzymatiques sont décelables dès la vie embryonnaire chez le Raton et le Iapereau (Bradshaw, I968 ; Corring et Aumaitre, I970 ; Courtot et al., I970) ;

- elles présentent d'importantes variations pendant la vie post natale chez le Rat (Corring et Aumaitre, I970), chez le Lapereau (LEBAs et al., I97I), chez le Porcelet (WALkER, I959; HartMan et al., I96r; Corring et AumaITre, i97I).

Entre la naissance et le sevrage se produit un important bouleversement physiologique dû au changement de la nature de l'alimentation : le jeune animal passe d'un régime uniquement lacté à un régime solide concentré. Or, au cours d'un premier travail sur le Lapereau (LEBAS et al., I97I), nous avons montré que :

- 1'équipement enzymatique du pancréas présente une brusque augmentation des activités entre le $22^{\mathrm{e}}$ et le $24^{\mathrm{e}}$ jour ;

- la quantité de matière sèche ingérée par $\mathrm{kg}$ de poids vif augmente à partir du $\mathrm{I} 8-20^{\circ}$ jour, par suite de la consommation d'aliment complémentaire solide.

La question posée dans ce travail est de savoir si les variations observées sont conformes à celles enregistrées chez l'animal adulte, c'est-à-dire à une adaptation de la glande pancréatique au régime alimentaire. (REBoud et al., I962 ; Bucko et KOPEC, I968). Les résultats obtenus ne permettent pas d'envisager une interprétation causale. L'augmentation des activités enzymatiques peut être due, soit à l'alimentation, soit à un facteur lié à l'âge (ou au poids), soit aux deux facteurs simultanément.

Pour essayer de lever cette ambiguité, nous avons procédé, dans l'expérience décrite ici, à un sevrage brutal réalisé à des dates variables, entraînant un changement quasi instantané de la nature de l'aliment.

\section{MATÉRIEL, ETT MÉTHODES}

\section{Les animaux}

L'expérience a porté sur II4 lapereaux issus de I4 portées, de race Californienne, nés le même jour et abattus en 9 stades répartis avant et après le sevrage. Les animaux ont été répartis en 2 lots homogènes de 7 portées. Un premier lot a été sevré au $2 \mathrm{I}^{\mathrm{e}}$ jour d'âge et le second au $30^{\mathbf{e}}$ jour. Les conditions d'élevage ont été identiques à celle décrites dans le travail précédent (LeBAs et al., r971).

\section{Régimes expérimentaux}

Jusqu'à 2 I jours pour le Ier lot et 30 jours pour le second, les lapereaux n'ont reçu que du lait de lapine. De manière à obtenir une croissance proche de la normale, et éliminer ainsi l'influence propre du poids des animaux, les portées sevrées à $3^{\circ}$ jours ont été allaitées par 2 lapines 
entre le $2 \mathrm{I}^{\mathrm{e}}$ et le 30 jour. Durant toute la période d'allaitement, les portées ont été maintenues à effectif constant ( 9 par portée jusqu'à 7 jours et 8 ensuite) grâce au remplacement des sujets abattus par des lapereaux élevés dans les mêmes conditions. A compter de la date du sevrage (2I ou 3o jours), les animaux n'ont eu à leur disposition qu'un aliment concentré (LEBAS, I968).

\section{Abattages et prélèvements}

Les animaux ont été sacrifiés à l'âge de : 7, I6, 21, 24, 28, 30, 32, 36 et 43 jours, compte tenu des observations réalisées lors de la premiére expérience (LEBAs $e t$ al., I97 I). Dix lapereaux ont été sacrifiés à chacun des trois premiers stades et 7 lapereaux par lot aux stades suivants. Les animaux abattus au $2 \mathrm{I}^{\mathrm{e}}$ jour n'avaient pas consommé d'aliment concentré.

Pour chacun des stades d'abattage, il a été prélevé un lapereau par portée de manière à éliminer un éventuel effet " portée ". Les contrôles avant sacrifice (mise à jeun, pesée) sont identiques à ceux décrits dans le premier travail (LeBAs et al., 197I).

\section{Dosages}

Immédiatement après abattage, le pancréas est extrait, pesé frais et homogénéisé à l'aide d'un ultra-turrax Staufen type TP I $8 / 2$ dans l'eau distillée glacée. Après homogénéisation des pancréas, il est procédé sur le broyat aux dosages des protéines totales et des activités enzymatiques de la trypsine, chymotrypsine, lipase et amylase. Les techniques et les modes d'expression sont ceux décrits par Corring et Aumaitre (1970).

\section{RÉSULTATS}

Dans le but de simplifier la présentation des résultats, nous désignerons les lapereaux nourris du lait maternel jusqu'au $30^{\mathrm{e}}$ jour par la lettre $\mathrm{L}$ et les lapereaux sevrés au 2 I e jour par la lettre $S$.

\section{Évolutions pondérales}

Pour tous les animaux, 1'augmentation du poids vif est régulière durant les 3 premières semaines. La croissance des lapereaux $L$ s'accélère entre le $2 I^{\mathrm{e}}$ et le $3^{\circ}$ jour et devient plus importante par la suite (fig. I). I a croissance des lapereaux $S$ subit un arrêt et une reprise entre le $2 \mathrm{I}^{\mathrm{e}}$ et $28^{\mathrm{e}}$ jour pour devenir identique à celle des animaux $\mathrm{L}$, en fin d'expérimentation (fig. I).

En ce qui concerne le développement pondéral du pancréas (fig. I), nous notons que les valeurs obtenues sur les lapereaux $S$ après le $26^{\mathrm{e}}$ jour et jusqu'au $32^{\mathrm{e}}$ jour sont significativement supérieures à celles enregistrées sur les animaux L. L'écart n'est plus significatif par la suite, bien que le poids du pancréas des lapereaux $\mathrm{S}$ demeure supérieur.

L'étude de l'évolution du logarithme du poids frais du pancréas ( $\mathrm{Y}$ ) en fonction de celui du poids vif $(\mathrm{X})$ (fig. 2 ) permet de définir une première droite entre le $\eta^{\mathbf{e}}$ et $2 I^{\mathrm{e}}$ jour dont l'équation est :

$$
\mathrm{Y}_{0}=0,62 \mathrm{X}_{0}+0,99
$$

A partir du $24^{\mathrm{e}}$ jour, on obtient pour chacun des deux régimes une droite d'équation différente

$$
\begin{aligned}
& \mathbf{Y}_{\mathbf{L}}=\mathrm{I}, 066 \mathrm{X}_{\mathrm{L}}-0,04 \\
& \mathbf{Y}_{\mathrm{S}}=\mathrm{I}, 0 \mathrm{or} 3 \mathrm{X}_{\mathrm{S}}+0, \mathrm{I} 7
\end{aligned}
$$


Les 2 droites sont de pente identique, mais diffèrent significativement par leur ordonnée à l'origine. Ainsi, le développement relatif du pancréas est marqué par une phase d'allométrie minorante jusqu'au $2 \mathrm{I}^{\mathbf{e}}$ jour $(\mathrm{P}<0, \mathrm{OI})$, puis par une phase

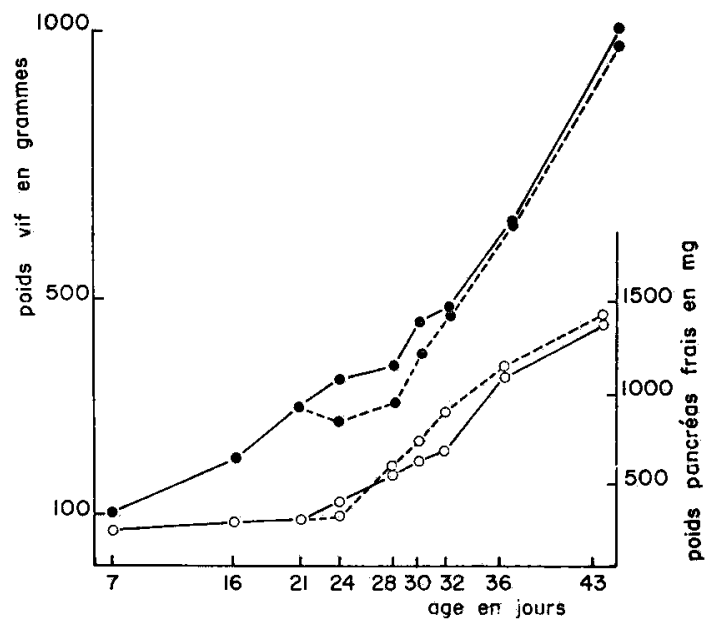

FIG. I. - Évolution du poids vif et du pancréas avec l'âge et en fonction du sevrage

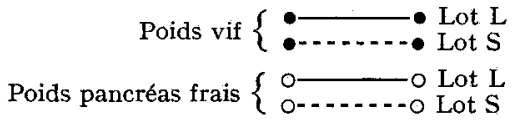

d'isométrie quel que soit le régime. Cependant, le poids du pancréas des lapereaux $\mathrm{S}$ est significativement plus élevé que celui des lapereaux $L$, pour un même poids vif à partir du $24^{\mathrm{e}}$ jour.

\section{Quantités d'aliments consommées}

Dans le but de pouvoir comparer entre eux les ingesta, nous avons exprimé les consommations de lait et d'aliment concentré moyennes par lapereau, par les quantités de matière sèche correspondantes (tabl. I).

Il apparaît que les quantités consommées par lapereau, quel que soit le régime, soient identiques jusqu'au $25^{\mathrm{e}}$ jour. Entre les périodes 22-25 jours et 26-29 jours, les quantités de matière sèche consommées augmentent faiblement pour les animaux I. ( 5 p. Ioo) et fortement pour les lapereaux S ( I I0 p. IOo). Pour les detux lots expérimentaux, les quantités de matière sèche consommées doublent dans les 4 jours qui suivent le sevrage par rapport aux 4 jours qui le précèdent. Enfin, les consommations deviennent identiques pour les 2 régimes à partir du $34^{\mathrm{e}}$ jour.

\section{Activités enzymatiques du pancréas}

\section{Activités enzymatiques totales.}

L'examen des activités enzymatiques totales reportées sur les figures 3 et 4 met en évidence deux types de résultats concernant la stimulation du pancréas, d'une part, l'ampleur de la réponse à cette stimulation, d'autre part. 


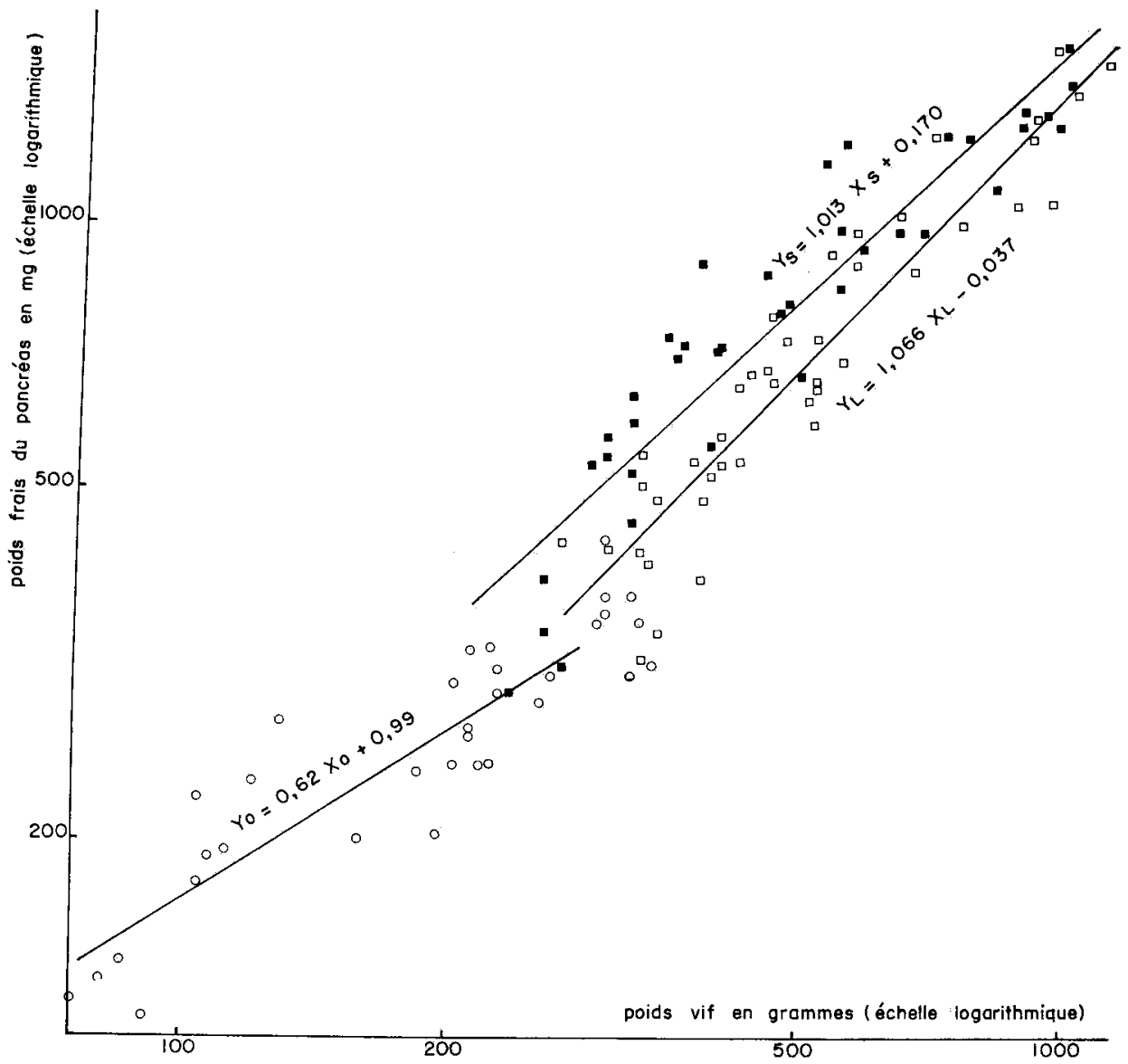

FIG. 2. - Évolution pondérale du pancréas frais en fonction du poids vif chez le Lapin

$$
\left\{\begin{array}{l}
0: \text { Ensemble des animaux entre } 7 \text { et } 2 \text { I jours } \\
\text { a: Lapereaux } \mathrm{S} \\
\text { : Lapereaux } \mathrm{L}
\end{array}\right.
$$

TABLEAU I

\begin{tabular}{|c|c|c|c|c|c|c|c|c|c|}
\hline \multirow{2}{*}{ Traitements } & \multirow{2}{*}{ Aliment } & \multicolumn{8}{|c|}{ Stades en jours } \\
\hline & & $1-8$ & $9-17$ & $18-21$ & $22-25$ & $26-29$ & $30-33$ & $34-37$ & $38-42$ \\
\hline \multirow{2}{*}{ Lot $L\left({ }^{1}\right)$} & Lait & 4,81 & 6,81 & 7,86 & 15,41 & 16,18 & - & - & - \\
\hline & Aliment concentré & - & 一 & - & - & - & 37,83 & 70,21 & 86,15 \\
\hline \multirow{2}{*}{ Lot S $\left({ }^{2}\right)$} & Lait & 4,17 & 6,32 & 7,71 & $\longrightarrow$ & 一 & - & - & - \\
\hline & Aliment concentré & - & - & - & 16,18 & 41,07 & 60,25 & 72,01 & 84,54 \\
\hline
\end{tabular}

Quantités consommées (matière sèche) en g/j et par lapereau

(1) Lapereaux sevrés au $30^{\mathrm{e}}$ jour.

(2) Lapereaux sevrés au $21^{\mathrm{e}}$ jour: 
- Pour les 2 régimes, les activités de l'amylase, de la lipase et de la chymotrypsine augmentent fortement après le $2 \mathrm{I}-24^{\mathrm{e}}$ jour (fig. 3 et 4 ). Par contre, l'augmentation de l'activité trypsique est plus tardive, vers 32 jours (fig. 4) et ses variations ne sont significatives qu'entre 32 et 36 jours, pour les 2 régimes.

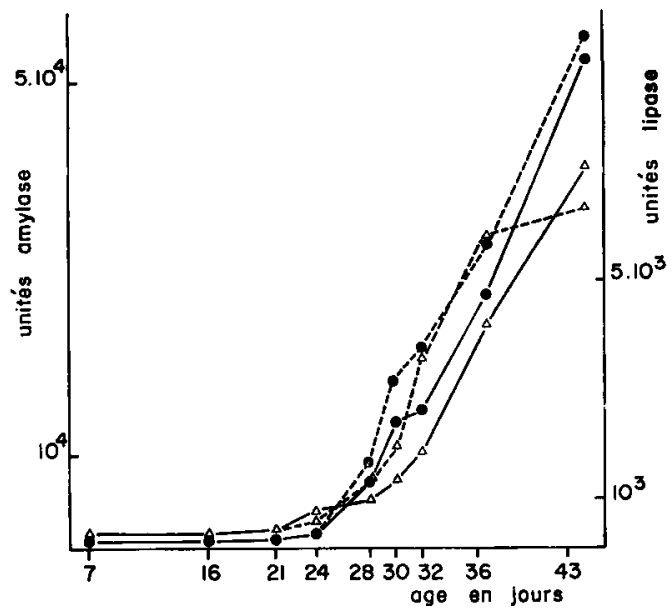

FIG. 3. - Activites enzymatiques totales de la lipase et de l'amylase
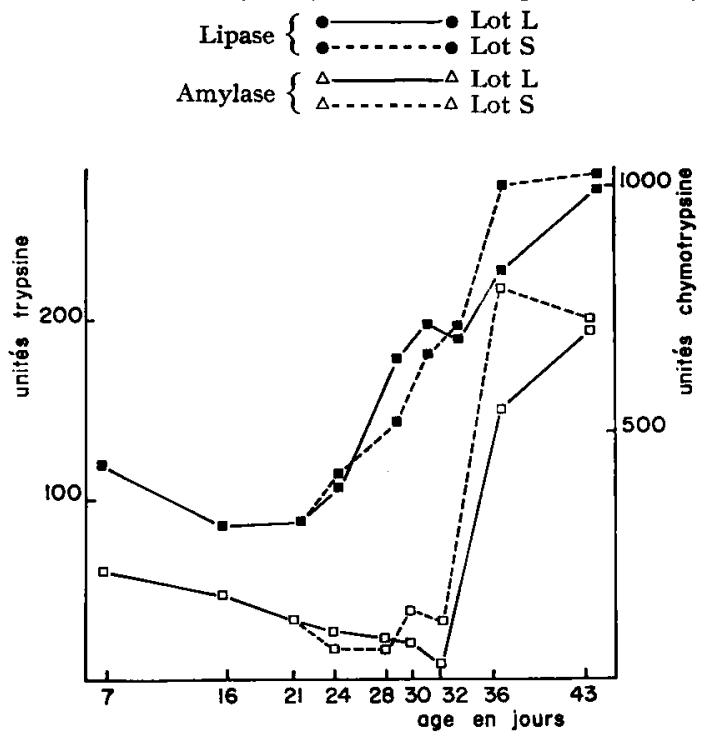

Fig. 4. - Activites enzymatiques totales de la trypsine et de la chymotrypsine

$$
\begin{aligned}
& \text { Trypsine }\left\{\begin{array}{l}
\square-\ldots \text { Lot } L \\
\square-\ldots \text { Lot } S
\end{array}\right. \\
& \text { Chymotrypsine } \begin{cases}\square & \text { Lot } L \\
\cdots \ldots \ldots & \text { Lot } S\end{cases}
\end{aligned}
$$

- Les activités lipasique et amylasique ont tendance, dès le $28^{\mathrm{e}}$ jour, à être supérieures chez les lapereaux $\mathbf{S}$; toutefois, la différence n'est significative qu'au $3^{e}$ jour. Quant aux activités trypsique et chymotrypsique, le phénomène observé 


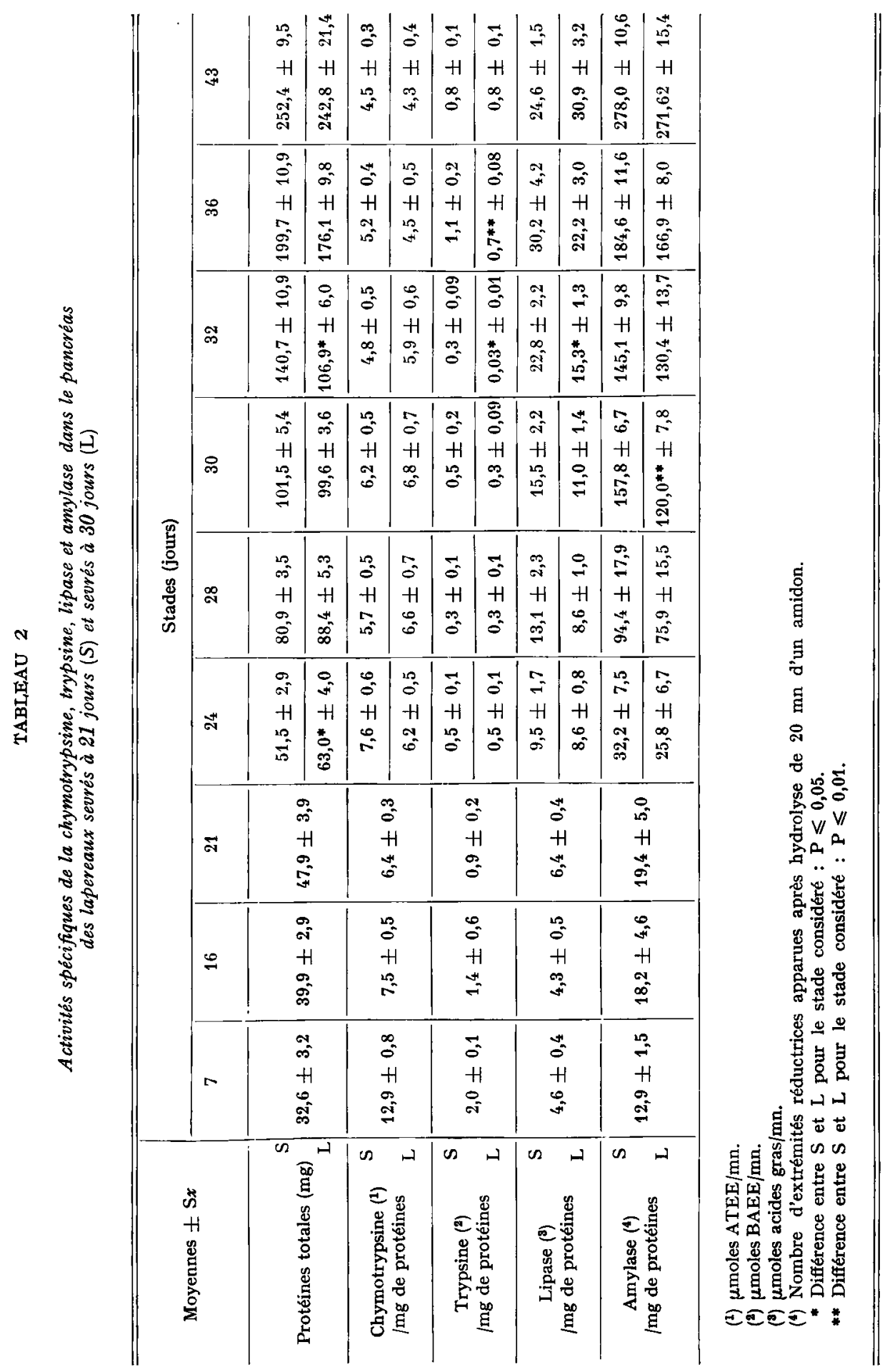


semble plus complexe. En effet, 1'activité chymotrypsique semble plus importante chez les animaux $\mathrm{L}_{\mathrm{H}}$ jusqu'au $3^{\circ}$ jour, date de sevrage de ces derniers. Passé ce stade, elle devient inférieure à celle déterminée chez les animaux S. Toutefois, pour aucun des stades considérés, les différences ne sont significatives.

L'activité trypsique, dans le pancréas des lapereaux $\mathrm{S}$, marque une diminution par rapport à celle des animaux $\mathrm{I}$ dès le $24^{\mathrm{e}}$ jour (différence non significative). puis devient significativement supérieure après le $30^{\mathrm{e}}$ jour et jusqu'au $3^{\mathrm{e}}$ jour inclus.

Par ailleurs, il importe de noter qu'il n'a pas été possible de détecter une activité trypsique pour un animal du régime $S$ et pour quatre animaux du régime $L$, au $3^{\mathrm{e}}$ jour.

\section{Activités enzymatiques spécifiques.}

Lorsque 1'on exprime les activités enzymatiques par $\mathrm{mg}$ de protéines totales (tabl. 2), nous retrouvons une évolution de la lipase et de l'amylase identique à celle décrite avec les activités totales correspondantes, à savoir une augmentation des valeurs après le $2 \mathrm{r}-24^{\mathrm{e}}$ jour. Par contre, le comportement des enzymes protéolytiques est différent.

L'activité spécifique de la chymotrypsine diminue fortement jusque vers le

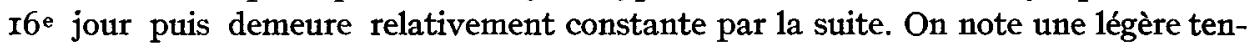
dance à la diminution durant cette dernière période, bien que les différences ne soient pas significatives.

Quant à l'activité trypsique, elle diminue également jusqu'au $\mathrm{I} 6^{\mathrm{e}}$ jour chez les animaux $L$, elle demeure sensiblement constante $d u\left(6^{\mathrm{e}}\right.$ au $30^{\mathrm{e}}$ jour, puis diminue fortement entre le $30^{\mathrm{e}}$ et le $32^{\mathrm{e}}$ jour pour augmenter ensuite et atteindre des valeurs comparables à celles notées au cours de la $3^{e}$ semaine. Chez les animaux $\mathrm{S}$, nous notons une évolution comparable, mais avec moins d'amplitude entre les valeurs. Seules les différences aux $32^{\mathrm{e}}$ et $3^{6^{\mathrm{e}}}$ jour entre les régimes $S$ et $\mathrm{L}$ sont significatives et montrent que l'activité trypsique est supérieure chez les lapereaux $\mathbf{S}$.

\section{DISCUSSION}

\section{Évolutions pondérales}

Nous retrouvons, dans cette expérience, une croissance comparable à celle que nous avions observée précédemment (LEBAS et al., I97I) sur des animaux de la même souche, les 3 premières semaines tout au moins. En effet, les conditions expérimentales entraînent, pour le lot $\mathrm{S}$, un choc au sevrage (2I jour) marqué par un arrêt momentanée de la croissance. Ce phénomène ne se retrouve pas avec les animaux $\mathrm{L}$, ce qui est certainement dû au fait que, au moment imposé pour le sevrage (30 jour), les lapereaux L sont plus matures. Ennfin, il faut souligner qu'à partir du $24^{\mathrm{e}}$ jour, le poids vif des animaux des 2 lots est toujours inférieur à celui des lapereaux élevés dans des conditions classiques d'élevages, précédemment utilisées (LEBAS et al., r97I). Cela est dû au sevrage précoce pour les animaux du lot S. Pour ceux du lot L, la faible production laitière des lapines (fin de lactation) et l'absence d'aliment complémentaire entre 20 et 30 jours sont à l'origine de cette croissance plus faible. 
En ce qui concerne le développement pondéral du pancréas, nous retrouvons une évolution relative du poids de la glande identique à celle déjà observée (LEBAS et al., r97I). Toutefois, le changement d'allométrie est situé à un âge apparemment plus tardif (22 contre I4 jours). Cette variation doit être attribuée plus à un nombre différent de stades utilisés qu'à un autre phénomène. Il faut préciser que ce changement d'allométrie, par contre, se situe dans une même gamme de poids vif.

A partir du $24^{\mathrm{e}}$ jour, les lapereaux $\mathrm{S}$ ont un pancréas relativement plus lourd (pour le même poids vif) que ceux du lot $\mathrm{L}$. Il semble que le choc au sevrage affecte plus la croissance corporelle globale que celle du pancréas. Cette différence semble acquise, jusqu'au $43^{\mathrm{e}}$ jour tout au moins.

\section{Activités enzymatiques}

De l'ensemble des résultats, il ressort que, quel que soit le régime imposé, nous retrouvons une évolution des activités enzymatiques, totales et spécifiques, identique à celle décrite sur les lapereaux élevés dans des conditions classiques de sevrage (LEBAs et al., I97I). En ce qui concerne les activités protéolytiques spécifiques, nous avons montré ici qu'elles diminuent jusqu'au I $^{\mathrm{e}}$ jour, puis demeurent constantes par la suite. Dans notre premier travail, cette diminution s'est poursuivie jusqu'au. II ${ }^{\mathbf{e}}$ jour seulement. La différence observée s'explique, comme le changement d'allométrie du pancréas, simplement par le fait que dans les deux expériences le nombre de stades d'abattages n'est pas le même. Dans ce travail, en effet, nous avions étudié l'évolution des différents critères principalement durant les 3 premières semaines de vie, alors qu'ici nous avons utilisé, pendant cette période, 3 stades seulement contre 7 stades dans la première expérience (LEBAs et al., I97I).

Dans le cas du présent travail, la durée de l'expérimentation (43 jours contre 32 jours) nous a permis, également, de mettre en évidence une évolution particulière de l'activité trypsique entre le $3^{\mathrm{e}}$ et le $43^{\mathrm{e}}$ jour. Celle-ci se manifeste par une augmentation des valeurs exprimées par $\mathrm{mg}$ de protéines, particulièrement dans le cas du lot L.

En ce qui concerne la réponse plus ou moins marquée de certaines enzymes en fonction des différents régimes, l'expérience n'a pas mis en évidence de phénomène très simple.

L,es activités amylasique et lipasique (activités totales et spécifiques) apparaissent toujours supérieures dans le cas des lapereaux $S$, mais la différence entre les 2 lots n'est significative qu'au $3^{2}$ jour. Cette date correspond, pour les lapereaux $\mathrm{L}$, à la manifestation maximale du choc au sevrage réalisé deux jours plus tôt. Parallèlement, à 32 jours, l'activité de la chymotrypsine chez les animaux $L$ marque un arrêt dans son accroissement d'un stade de contrôle à l'autre. Si le choc au sevrage à 30 jours se manifeste très peu sur les croissances pondérales (Poids vif, Pancréas), il semble, par contre, affecter le pouvoir de synthèse de la glande pancréatique. Cela semble confirmé par l'activité trypsique par mg de protéines ro fois plus faible à 32 jours qu'à 30 , pour les lapereaux L.

Enfin, le sevrage à 2I jours des animaux $\mathbf{S}$ n'a pas entraîné de modification apparente des activités enzymatiques du pancréas. Cela est probablement dû au bas niveau d'activité de la glande. 
Cette expérience a nettement mis en évidence le fait que, quel que soit la date du sevrage des animaux, donc de la variation du régime alimentaire, l'équipement enzymatique du pancréas exocrine est fortement stimulé à un âge fixe qui se situe entre le $2 \mathrm{I}^{\mathrm{e}}$ et $24^{\circ}$ jour. A cet âge existe donc un facteur indépendant de l'alimentation et responsable de cette stimulation.

La nature de ce facteur ne peut être déterminée ici, mais on peut suggérer que ce facteur puisse être de type hormonal, lié au développement physiologique du jeune animal.

Les travaux de Prochazka et al. (I964) sur le Rat, et récemment de CourToT (I97I) sur le Lapereau, supportent une telle hypothèse. En effet, l'injection d'une hormone corticoïde avant l'augmentation naturelle de l'activité amylasique chez ces animaux, provoque cette variation. Ia même injection effectuée après l'augmentation naturelle de l'activité enzymatique demeure sans effet, l'animal semble être pourvu en quantité suffisante de cette hormone et un excès n'entraîne aucune modification.

Tout paraît se passer comme si une apparition ou une augmentation du facteur considéré ( $15-18$ e jour chez le Rat, $2 \mathrm{I}_{-2} 4^{\mathrm{e}}$ jour chez le Lapereau) déclenchait ou stimulerait l'activité sécrétoire du pancréas exocrine.

L'influence du facteur hormonal sur l'activité pancréatique a également été mise en évidence par Kuı,KA et DuKsin (I967), YALOWSKI et al. (I969).

Enfin, ce travail met en évidence une influence de la composition du régime alimentaire, bien qu'elle ne soit que rarement significative. Chez les lapereaux sevrés au $2 \mathrm{I}^{\mathrm{e}}$ jour les activités enzymatiques sont en général supérieures à celles déterminées dans le pancréas des animaux sevrés au $30^{\mathbf{e}}$ jour. De plus, chez ces derniers, l'activité enzymatique subit à son tour une stimulation lors du changement de régime. Ces derniers résultats sont en faveur de l'hypothèse de l'adaptation du pancréas au régime alimentaire (GROSSMAN et al., I942; DESNUELI,E et al., I962 ; SNOOK et MEYER, I964).

\section{CONCLUSION}

Cette expérience, entreprise pour répondre aux questions qui se sont posées au terme d'un premier travail, a permis de mettre en évidence les faits importants suivants :

- durant la vie post natale du lapereau, il existe un âge précis où, même en l'absence de variation du mode d'alimentation, l'équipement enzymatique du pancréas exocrine est fortement stimulé ;

- l'ampleur de la réponse à cette stimulation semble ensuite légèrement modulée en fonction du régime alimentaire. Elle paraît en tout cas sensible à un phénomène complexe comme un sevrage brutal à 30 jours. 


\section{SUMMARY}

\section{CONFIRMATION OF THE EVOLUTION \\ OF THE EXOCRINE PANCREAS ENZYMATIC SYSTEM \\ IN THE RABBIT FROM BIRTH TO 6 WEEKS}

To confirm the hypothesis that the exocrine pancreas in young suckling rabbits adapts to variations in the diet at weaning, 2 lots of 57 rabbits were fed with the following experimental diets : diet.

I. Maternal milk only between birth and day $3^{\circ}$, then sudden weaning with a starch diet.

2. Maternal milk only between birth and day $2 \mathrm{I}$, then sudden weaning with the same satrch

Trypsine, chymotrypsine, lipase, and amylase enzymatic activities were determined at 9 stages between day 7 and day 43 . The results show that no matter what the experimental diet was, all enzymatic activities increase about day 21 to day 24 independently of the animals' weight. These activities are slightly higher in animals weaned at $2 \mathrm{I}$ days. This seems to indicate that the feed is not the factor stimulating pancreatic enzyme development. However, it could possibly modify the amount of response to this stimulation.

\section{RÉFÉRENCES BIBLIOGRAPHIQUES}

BRADSHAW W. S., I968. Studies on pancreatic lipase : multiplicity distribution and development regulation. $\mathrm{Ph}$. D. Thesis, University of Illinois.

Bučko A., Kopec Z., Ig68. Adaptation of enzymes activity of the rat pancreas on altered food intake. Nutritio et Dieta., 10, 276-287.

Corring T., Aumaitre A., r97o. Mise en place et évolution de l'équipement enzymatique du pancréas exocrine du jeune Rat pendant la période embryonnaire, l'allaitement et le sevrage. $A n n$. Biol. anim. Bioch. Biophys. 10, 43-44.

Corring T., Aumaitre A., I97I in Development de quelques fonctions physiologiques après la naissance du porcelet par Aumaitre A. Journée du Porc de Vittel, 1971, p. ir-I2.

Courtot D., rg7r. Données non publiées.

Courtot D., Corring T., Laviouette P., 1970. Détection des proenzymes protéolytiques du pancréas du Lapin par immunofluorescence (embryon, nouveau-né, adulte). Ann. Biol. anim. Bioch. Biophys. 10, 29r-299.

Desnuelle P., Reboud J.-P., Ben Abdeljlil A., r962. Influence of the composition of the diet on the enzymes content of rat pancreas, in Ciba Foundation Symposium of the exocrine pancreas ; Churchill (London) p. 90.

Grossman M. I., Greengard H., Ivy A. C., 1942. The effect of dietary composition on pancreatic enzymes. Amer. J. Physiol. 138, 676-682.

Hartman P. A., Hays V.M., Baker R. C., Neagle L. H., Catrone D. W., i96r. Digestive enzyme development in the young pig. J. Anim. Sci. 20, Ir4-123.

Kutka R. G., Duksin D., 1964. Patterns of growth and $\alpha$-amylase activity in the developing chick pancreas. Biochim. Biophys. Acta, 91, 506-514.

Lebas F., I953. Mesure quantitative de la production laitière chez la Lapine. Ann. Zootech., 17, I68182 .

Lebas F., Corring T., Courtot D., i97r. Équipəment enzymatique du pancréas exocrine chez le Lapin, mise en place et évolution de la naissance au sevrage, relation avec la composition du régime alimentaire. Ann. Biol. anim. Bioch. Biophys. 3, 399-413.

Prochazka P., Hahn P., Koldovsky D., Noh YNeK M., Rokos J., r964. The activity of $\alpha$-amylase in homogenates of the pancreas of rats during early post-natal development. Physiol. Bohemoslov. 13, $288-29 I$.

Reboud J.-P., Ben Abdeljlit A., Desnuelle P., i962. Variation de la teneur en enzymes du pancréas du Rat en fonction de la composition des régimes. Biochim. Biophys. Acta, 58, 326-337.

SNOOK J. T., MEYER J. H., 1964. Responses of digestive enzymes to dietary protein. J. Nutr. 82 408-4I3.

WaLker D. M., 1959. The development of the digestive enzymes of the young animal. 1I. Carbohydrase enzymes development in the young pig. J. Agric. Sci. 52, 357-37o.

YALOWSKI U., Zelikson R., KuLKa G., I969. The effect of hydrocortisone on the accumulation of amylase in embryonic chick pancreas. Febs. Letters, 2, 323-326. 